\title{
Analytical Aberration-Corrected STEM of Ferroelectric Functional Interfaces
}

\author{
Q.M. Ramasse ${ }^{1}$, M. Arredondo ${ }^{2}$, M. Weyland ${ }^{3}$, R. Mahjoub ${ }^{2}$, I. Vrejoiu ${ }^{4}$, D. Hesse ${ }^{4}$, N.D. Brown- \\ ing $^{5,6}$ M. Alexe ${ }^{4}$, P. Munroe ${ }^{2}$ and V. Nagarajan ${ }^{2}$
}

${ }^{1}$ SuperSTEM Laboratory, STFC Daresbury, Keckwick Lane, Daresbury WA4 4AD, U.K.

${ }^{2}$ School of Mat. Sci. and Engineering, U. of New South Wales, Sydney NSW 2052, Australia.

${ }^{3}$ Monash Centre for Electron Microscopy, Monash University, Victoria 3800, Australia.

${ }^{4}$ Max Planck Institute for Microsctructural Physics, Weinberg 2, D-06120 Halle, Germany

${ }^{5}$ Dept. of Chem. Eng. and Mat. Sci., University of California-Davis, Davis CA 95616

${ }^{6}$ Lawrence Livermore National Laboratory, 7000 East Avenue, Livermore CA 94550

In recent years progress in electron microscopy has pushed resolution to sub- $\AA$ values at $300 \mathrm{kV}$ and below [1,2]. As an essential corollary to these instrumentation developments, significant increases in detection limits and signal-to-noise ratios were achieved such that the improved data collection ability and greater flexibility of the instruments have arguably proved the most beneficial advances for the materials sciences community, allowing users to utilise efficiently all available analytical signals. These benefits are particularly evident in the specific example of the chemical structure of dislocation cores in ultra-thin ferroelectric $\mathrm{PbZr}_{0.2} \mathrm{Ti}_{0.8} \mathrm{O}_{3}$ oxide films [3]. The current trend of aggressive downsizing of the building blocks of silicon electronics has resulted in the functional material being confined to nanometric size volume and the presence of even a single defect and its associated longrange field could indeed adversely affect the performance and/or reliability of the device. Given the complex chemistry of the ferroelectric oxides involved, the vital and still largely unanswered question of how a dislocation core impacts the nanoscale local chemistry of the functional interface can only be addressed using a combination of analytical techniques and instrument settings. High acceleration voltage and ultra-fine probes are required for high-resolution imaging (Fig. 1a), lower, lessdamaging voltages and fine probes can provide atomically-resolved EELS information (Fig. 2a), while lower voltage and slightly larger probes can be used for high-resolution EDS work essential for the localisation of heavy metals such as $\mathrm{Pb}$ and $\mathrm{Ru}$ (Fig. 2b). This combination of techniques thus reveals $\mathrm{O}$ deficiency within the defect cores as well as cation non-stoichiometry in the vicinity of the dislocations. Additionally, the improvements in stability and signal-to-noise of the latest generation of aberration-corrected STEM instruments, along with much improved environmental control in microscope rooms, mean that strain mapping, a technique traditionally reserved to the processing of HREM images, can now yield quantitative data from Z-contrast images. Fig. 1b thus shows an example strain map in strong agreement with quantitative models that predict strain-assisted segregation of $\mathrm{Pb}$ around the dislocation cores.

In conjunction with fully quantitative image simulations and image analysis, this wealth of high signal-to-noise analytical signals can thus provide a full sample characterisation. It is possible to observe directly and quantitatively phenomena such as stress-assisted diffusion and Cottrell atmospheres, which thus far were only conjectured to occur for dislocations in functional oxide interfaces.

References

[1] C. Kisielowski, B. Freitag, M. Bischoff, et al, Microscopy and Microanal. 14, 454-462 (2008)

[2] H. Sawada, Y. Tanishiro, N. Ohashi, et al., J. Electron Microscopy 58, 357-361 (2009)

[3] M. Arredondo, Q.M. Ramasse, M. Weyland et al., Advanced Materials, in press (2010) 
[4] This work was carried out in part at the National Centre for Electron Microscopy, under the auspices of the U.S. Department of Energy under contract number DE-AC02-05CH11231.

a)

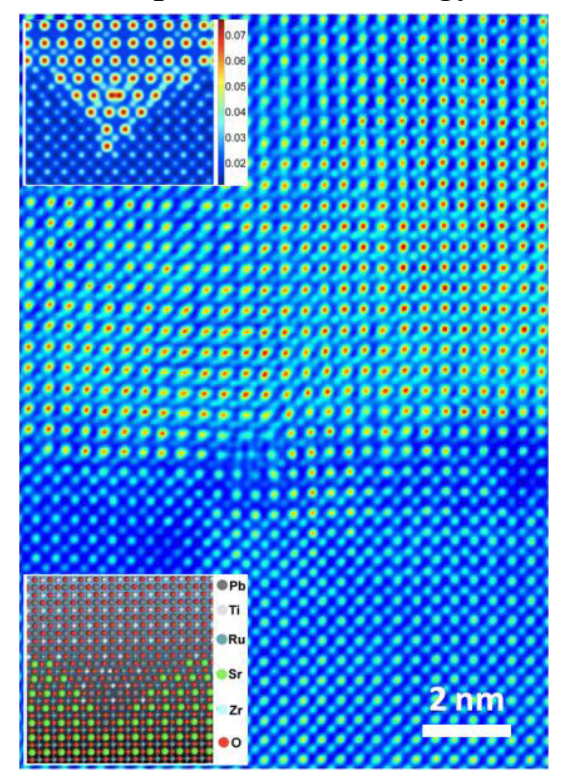

b)

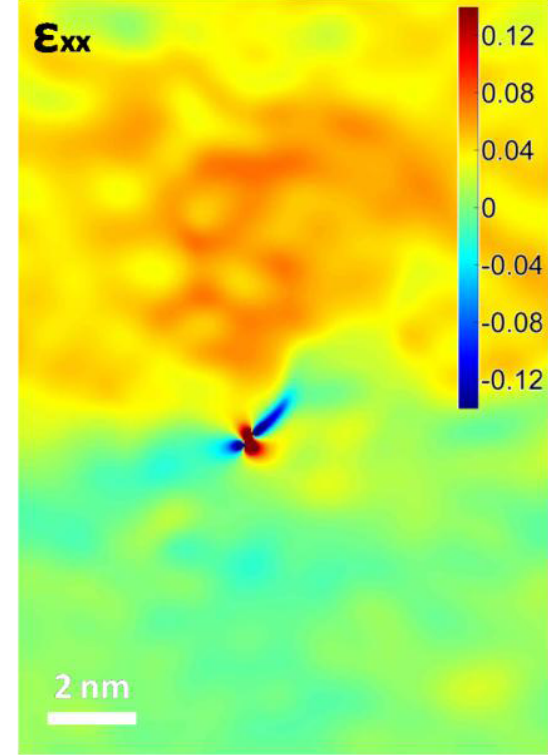

FIG. 1. a) Z-contrast image of an edge dislocation at the $\mathrm{PbZr}_{0.2} \mathrm{Ti}_{0.8} \mathrm{O}_{3}$ (functional ferroelectric, top) $/ \mathrm{SrRuO}_{3}$ (electrode, bottom) interface, recorded on the TEAM microscope [1] at $300 \mathrm{kV}$. For clarity, the image was processed using a maximum entropy probe deconvolution algorithm. Inserts show a model for the dislocation (bottom) and a quantitative multi-slice simulation of the structure, displayed on an identical colour scale (top). b) Corresponding strain map, showing the $\varepsilon_{\mathrm{xx}}$ component of the strain tensor relative to the SRO lattice at $0.9 \mathrm{~mm}$ spatial resolution obtained using geometrical phase analysis.
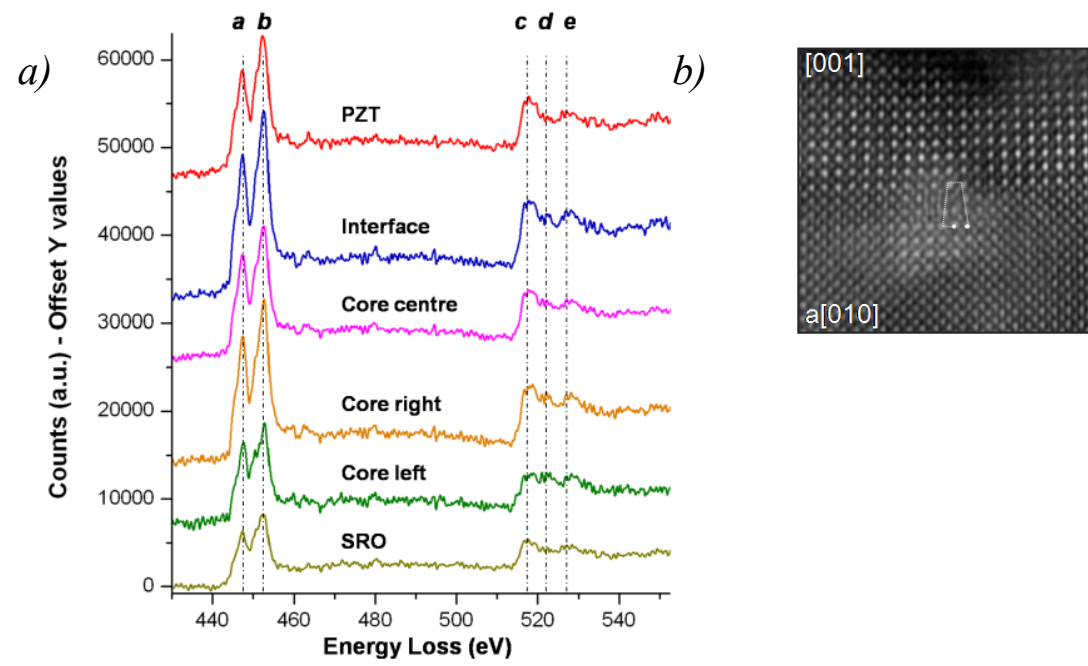

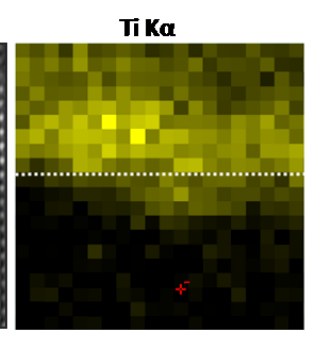

$\mathbf{P b} \mathbf{M a}$

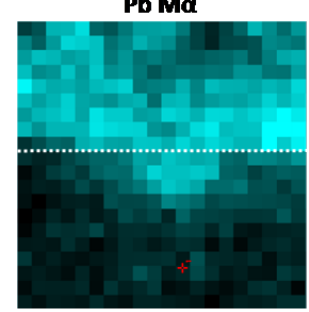

Ru Ka

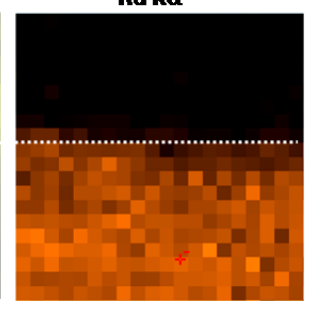

Sr Ka

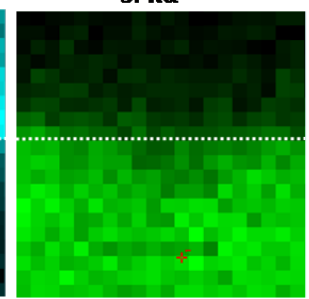

FIG. 2. a) Position-resolved EELS spectra acquired at $100 \mathrm{kV}$ on an aberration-corrected VG HB501, showing variations around the dislocation core of the $\mathrm{Ti} \mathrm{L}_{2,3}$ and $\mathrm{O} \mathrm{K}$ edges. The changing c/d peak ratio is attributable to changes in the linearity of the Ti-O-Ti bonds and therefore suggests some $\mathrm{O}$ deficiency around the core. b) Elemental EDS maps for $\mathrm{Ti}, \mathrm{Ru}, \mathrm{Pb}$ and $\mathrm{Sr}$ of the area represented on the top-left image. The micrograph was recorded at $300 \mathrm{kV}$ before the EDS analysis, which was carried out at $80 \mathrm{kV}$. There is clear evidence of $\mathrm{Pb}$ diffusion around the dislocation core into the $\mathrm{SrRuO}_{3}$ (the white dotted line marks the position of the interface). 